Article

\title{
Enhanced and Tunable Electrorheological Capability using Surface Initiated Atom Transfer Radical Polymerization Modification with Simultaneous Reduction of the Graphene Oxide by Silyl-Based Polymer Grafting
}

\author{
Erika Kutalkova $^{1}$, Miroslav Mrlik ${ }^{1, * \mathbb{C}}$, Marketa Ilcikova ${ }^{1,2,3}{ }^{\mathbb{D}}$, Josef Osicka ${ }^{1}{ }^{1}$, \\ Michal Sedlacik ${ }^{1}$ (1) and Jaroslav Mosnacek ${ }^{2,4}$ (1) \\ 1 Centre of Polymer Systems, University Institute, Tomas Bata University in Zlin, Trida T. Bati 5678, \\ 76001 Zlin, Czech Republic; ekutalkova@utb.cz (E.K.); marketa.ilcikova@savba.sk (M.I.); \\ osicka@utb.cz (J.O.); msedlacik@utb.cz (M.S.) \\ 2 Polymer Institute, Slovak Academy of Sciences, Dubravska cesta 9, 84541 Bratislava 45, Slovakia; \\ jaroslav.mosnacek@savba.sk \\ 3 Department of Chemistry, Lodz University of Technology, Institute of Polymer and Dye Technology, \\ 90924 Lodz, Poland \\ 4 Department of Polymer Engneering, Faculty of Technology, Tomas Bata University in Zlin, Vavreckova 275, \\ 76272 Zlin, Czech Republic \\ * Correspondence: mrlik@utb.cz
}

Received: 25 January 2019; Accepted: 13 February 2019; Published: 24 February 2019

\begin{abstract}
In this study, a verified process of the "grafting from" approach using surface initiated atom transfer radical polymerization was applied for the modification of a graphene oxide (GO) surface. This approach provides simultaneous grafting of poly(2-(trimethylsilyloxy)ethyl methacrylate) (PHEMATMS) chains and a controllable reduction of the GO surface. This allows the fine tuning of its electrical conductivity, which is a crucial parameter for applications of such hybrid composite particles in electrorheological (ER) suspensions. The successful coating was confirmed by transmission electron microscopy and Fourier-transform infrared spectroscopy. The molecular characteristics of PHEMATMS were characterized by gel permeation chromatography. ER performance was elucidated using a rotational rheometer under various electric field strengths and a dielectric spectroscopy to demonstrate the direct impact of both the relaxation time and dielectric relaxation strength on the ER effectivity. Enhanced compatibility between the silicone oil and polymer-modified GO particles was investigated using contact angle measurements and visual sedimentation stability determination. It was clearly proven that the modification of the GO surface improved the ER capability of the system due to the tunable conductivity during the surface-initiated atom transfer radical polymerization (SI-ATRP) process and the enhanced compatibility of the GO particles, modified by polymer containing silyl structures, with silicone oil. These unique ER properties of this system appear very promising for future applications in the design of ER suspensions.
\end{abstract}

Keywords: graphene oxide; (2-(trimethylsilyloxy)ethyl methacrylate), electrorheology; reduction; SI-ATRP

\section{Introduction}

Electrorheological (ER) fluids are smart materials that can change their mechanical properties upon the application of an external electric field. The ER fluids are generally heterogeneous systems 
mostly composed of solid particles and an insulating liquid medium [1,2]. After the application of an external electric field, the particles are polarized and start to create internal chain-like structures in the direction of the electric field (Scheme 1). This internal structure development leads to the transition from a liquid to a solid-like state accompanied by a viscosity increase in several orders of magnitude [3-5].

Graphene, one of the carbon allotropes, is a one atom thick carbon sheet consisting of a two-dimensional honeycomb lattice [6,7]. Due to its unique electrical and chemical properties, this material can be successfully utilized in many industrial applications, e.g., in the fabrication of electronic components, energy storage devices (super capacitors and lithium ion batteries), sensors, or drug delivery devices [8-10]. Chemical and physical functionalization of the graphene surface is necessary to achieve compatibility with its surroundings and thus several methods have been developed to improve the process of stabilization and the modification of the graphene $[9,11,12]$. One of them is the oxidation of the graphene by chemical methods, thus obtaining a graphene oxide (GO) with functional hydroxyl, carbonyl, carboxyl, or epoxy groups. These oxygen-containing groups are very important for the covalent bonding of other molecules [12,13]. The oxidation of graphene, however, leads to a very low electrical conductivity and poor ER performance due to the disruption of the conjugation structure of the graphene. However, for the ER application, the graphene needs to be oxidized to decrease its high conductivity, which, from the application point of view, is not desirable, as there is a risk of creating a short circuit [14-16]. Nevertheless, these drawbacks of GO can be overcome by an additional reduction of GO, which is usually performed before or after a suitable surface modification. The modification, consisting of grafting polymer chains onto the surface of GO particles, enables the control of its physicochemical properties [17]. The development of hybrid particles based on the GO and polymer coating can find utilization in various areas of applications, such as memory devices [18], flexible supercapacitors [19], or hydrogels for medical applications [20]. The most important benefit for such hybrid particles is the 2D shape of the GO and polymer coating, which provides excellent compatibility with its surroundings. As we recently demonstrated, a simultaneous reduction and coating process of GO led to both an increase of its conductivity, and a significant improvement of the sedimentation stability and ER performance thanks to its substantial shell, thus enhancing compatibility $[3,9,15,21]$. Similar results were published by other authors, however, in their study, the GO particles were non-covalently modified by various polymers, such as polyaniline [22] and polypyrrole [23], or covalently by silsesquioxane oligomers [24], and generally two steps were needed to receive the polymer coating and the appropriate conductivity for the ER investigation.

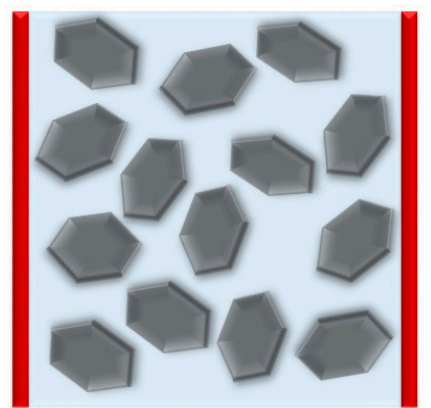

$E=0 \mathrm{kV} / \mathrm{mm}$

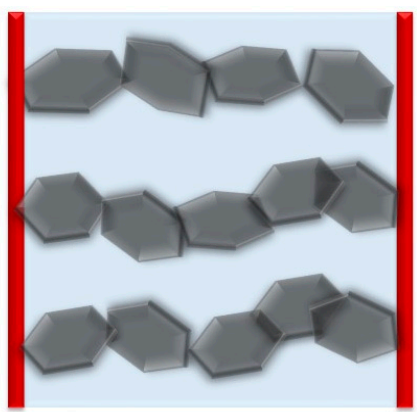

$E>0 \mathrm{kV} / \mathrm{mm}$

Scheme 1. Schematic illustration of the GO modified particles in the absence and in the presence of the external electric field.

This study deals with the modification of the GO particles achieved via surface-initiated atom transfer radical polymerization (SI-ATRP) of 2-(trimethylsilyloxy)ethyl methacrylate (HEMATMS). GO/poly(2-(trimethylsilyloxy)ethyl methacrylate) hybrids (GO-PHEMATMS) with two different molar masses of polymer were prepared with good control over the polymerization. Improved compatibility 
of the GO-PHEMATMS with silicone oil as a liquid medium as well as improved sedimentation stability were proven. The influence of conductivity on the ER performance was also demonstrated and a system with a uniquely high yield stress was obtained. Moreover, according to our knowledge, such a modification is a pioneer study, with regards to both the GO modification with silyl-based polymer grafting using the SI-ATPR approach, and its impact on the ER performance.

\section{Experimental Part}

\subsection{Materials}

Graphite (powder, $<20 \mu \mathrm{m}$, synthetic) as a precursor of $\mathrm{GO}$; sulfuric acid $\left(\mathrm{H}_{2} \mathrm{SO}_{4}\right.$, reagent grade, $95-98 \%)$, sodium nitrate $\left(\mathrm{NaNO}_{3}, \mathrm{ACS}\right.$ reagent, $\left.\geq 99 \%\right)$, potassium permanganate $\left(\mathrm{KMnO}_{4}, 97 \%\right)$, and hydrogen peroxide $\left(\mathrm{H}_{2} \mathrm{O}_{2}\right.$, ACS reagent, 29-32 wt. $\% \mathrm{H}_{2} \mathrm{O}_{2}$ basis) were used as oxidation agents for the preparation of GO sheets by a modified Hummers method as was described previously [25]. $\alpha$-Bromoisobutyryl bromide (BiBB, 98\%) was used as an initiator for the attachment onto the GO surface in the presence of triethyleneamine (TEA, $\geq 99 \%$ ) as a proton trap as described elsewhere [25]. Ethyl $\alpha$-bromoisobutyrate (EBiB, 98\%), $N, N, N^{\prime}, N^{\prime \prime}, N^{\prime \prime}$-pentamethyldiethylenetriamine (PMDETA, $\geq 99 \%$ ), copper bromide ( $\mathrm{CuBr}, \geq 99 \%)$, and anisole (99\%) were used as a sacrificial initiator, ligand, catalyst, and solvent, respectively, for ATRP of 2-(trimethylsilyloxy)ethyl methacrylate (HEMATMS, $99 \%$ ). All mentioned chemicals as well as diethyl ether (ACS reagent, anhydrous, $\geq 99 \%$ ) were purchased from Sigma Aldrich (St. Louis, MO, USA) and used as received except HEMATMS, which was passed through a short basic alumina column to remove a stabilizer. A mixture of polydimethyl siloxane (PDMS), Silgard 184, from Dow Corning (Midland, MI, USA) and dried silicone oil M200 from Lukosoil (Kolín, Czech Republic) was used as a polymer matrix. Tetrahydrofuran (THF, p.a.), dimethyl formamide (DMF, p.a.), acetone (p.a.), ethanol (absolute anhydrous, p.a.), toluene (p.a.), and hydrochloric acid ( $\mathrm{HCl}, 35 \%$, p.a.) were obtained from Penta Labs (Prague, Czech Republic) and used as received except THF, which was dried using flakes of sodium (99.9\%). Deionized water (DW) was used during all experiments.

\subsection{Surface Initiated ATRP}

The GO particles were prepared according to previously published papers [26]. Further, initiator immobilization was also performed according to our preceding study [25]. The immobilization onto the surface of GO was performed according to Scheme 2. The esterification reaction between the $\mathrm{OH}$ groups of $\mathrm{GO}$ and double-functional $\mathrm{BiBB}$ in the presence of triethylamine was carried out to obtain the initiator moiety covalently bonded on the surface of GO. The final SI-ATRP grafting procedure is also schematically shown in Scheme 2 and was performed as follows:
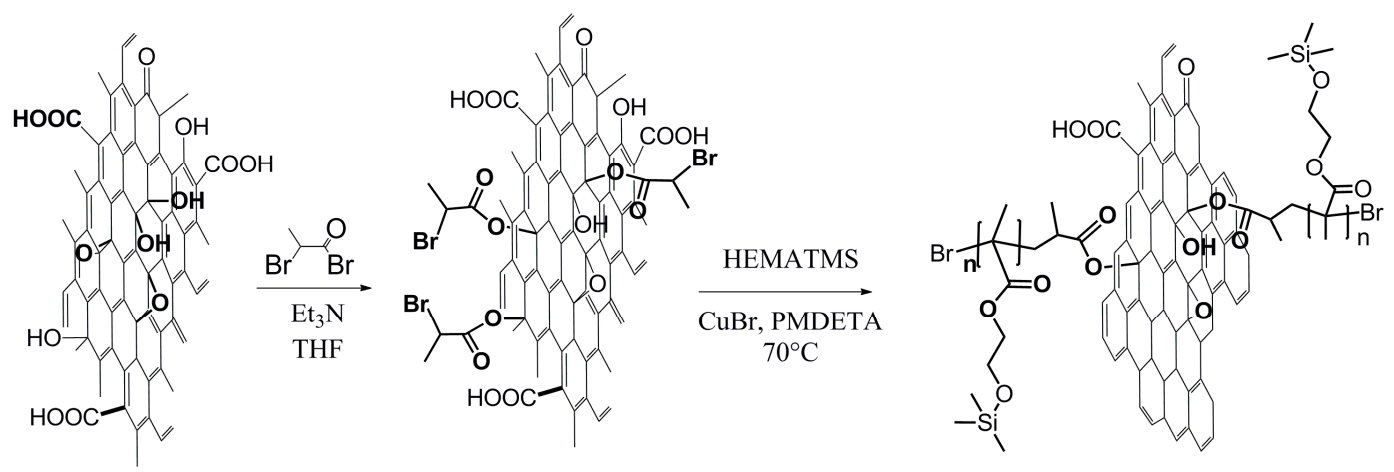

Scheme 2. Schematic illustration of the attachment of the ATRP initiator and grafting of the PHEMATMS from the GO surface with simultaneous partial reduction of the GO surface. 
GO sheets with an attached ATRP initiator $(1 \mathrm{~g})$ were put into a Schlenk flask and the flask was evacuated and backfilled with argon three times. HEMATMS (146.6 mmol, $32 \mathrm{~mL})$, EBiB (1.466 mmol, $0.215 \mathrm{~mL})$, PMDETA (5.864 mmol, $1.22 \mathrm{~mL})$, and anisole (32 mL) were pre-purged with argon, at least 10 minutes each, and added into the flask under argon flow. The system was degassed by three freeze-pump-thaw cycles, filled with argon, and CuBr $(1.466 \mathrm{mmol}, 0.2103 \mathrm{~g})$ was added to the frozen system under gentle argon flow. The molar ratio of the reactants [HEMATMS]:[EBiB]:[CuBr]:[PMDETA] was either [100]:[1]:[1]:[2] or [200]:[1]:[1]:[4] in order to receive the particles modified with PHEMATMS of the two different chain-lengths (marked later as GO-PHEMATMS-1 and GO-PHEMATMS-2, respectively) and differing also in electrical conductivity. Anisole was used as a solvent in a content of 50 vol.\%. The flask with the polymerization mixture was immersed into a $70{ }^{\circ} \mathrm{C}$ preheated silicone oil bath to initiate the polymerization process and the polymerization was performed under stirring at this temperature for 2.5 and 5 hours, respectively. Finally, the polymerization mixture was exposed to air to stop the polymerization. The conversions determined by proton spectra of nuclear magnetic resonance $\left({ }^{1} \mathrm{H}\right.$ NMR) spectroscopy (Bruker, Japan, $400 \mathrm{MHz}$ ) in $\mathrm{CDCl}_{3}$ were $67 \%$ and $60 \%$ for GO-PHEMATMS-1 and GO-PHEMATMS-2, respectively. The products, GO-PHEMATMS-1 and GO-PHEMATMS-2, were filtered, the particles in the form of filtration cake were redispersed in DMF (200 mL), and washed by acetone $(200 \mathrm{~mL})$ through the poly(tetrafluoro ethylene) PTFE filter paper with $0.44 \mu \mathrm{m}$. The redispersion and washing was performed twice and final cleaning and drying was done at the same time using diethyl ether (when the filtration cake was washed twice by $100 \mathrm{~mL}$ ). The molar mass and dispersity of the PHEMATMS chains were determined from the PHEMATMS initiated from the sacrificial initiator and present in the filtrate using GPC (Agilent, Tokyo, Japan) in THF at a flow rate of $1.0 \mathrm{~mL} \mathrm{~min}^{-1}$ using polystyrene standards.

\subsection{Particle Characterization}

Transmission electron microscopy (TEM) was performed using a JEM-2100Plus microscope (Jeol, Peabody, MA, USA) to evaluate the thickness and uniformity of the polymeric shell. Fourier transform infrared (FTIR) spectra were recorded on a Nicolet 6700 (Nicolet, USA) using an attenuated total reflectance (ATR) technique with Ge crystal and 64 scans with a resolution of $4 \mathrm{~cm}^{-1}$ within a wavenumber range of $4000-600 \mathrm{~cm}^{-1}$. The Raman spectra ( 3 scans, resolution of $2 \mathrm{~cm}^{-1}$ ) were collected on a Nicolet DXR (Nicolet, Midland, ON, Canada) using an excitation wavelength of $532 \mathrm{~nm}$. The integration time was $30 \mathrm{~s}$, while the laser power on the surface was set to $1 \mathrm{~mW}$. The powders were compressed to the form of pellets (diameter of $13 \mathrm{~mm}$, thickness of $1 \mathrm{~mm}$ ) by using a laboratory hydraulic press (Trystom Olomouc, H-62, Olomouc, Czech Republic). The electrical conductivity, $\sigma_{\mathrm{p}}$, measurements were performed on the same pellets as were used for Raman investigation, and measured using a Keithley 6517B (Keithley, Austin, TX, USA) multimeter and the four-point method. The contact angle measurement (CA) was evaluated from the static sessile drop method carried out on a Surface Energy Evaluation system equipped with a CCD camera (Advex Instruments, Brno, Czech Republic).

X-ray photo-electron spectroscopy (XPS) measurements were performed using a target factor analysis (TFA) XPS device from Physical Electronics. The base pressure in the XPS analysis chamber was approximately $6 \times 10^{-8} \mathrm{~Pa}$. The samples were excited by X-rays over a $400 \mu \mathrm{m}^{2}$ spot area with monochromatic $\mathrm{Al} \mathrm{K} \alpha_{1,2}$ radiation at $1486.6 \mathrm{eV}$. Photoelectrons were detected with a hemispherical analyser positioned at an angle of $45^{\circ}$ with respect to the normal to the sample surface. The energy resolution was approximately $0.5 \mathrm{eV}$. Survey-scan spectra were acquired at a pass energy of $187.85 \mathrm{eV}$, whereas for C 1s, individual high-resolution spectra were taken at a pass energy of $29.35 \mathrm{eV}$ and with a $0.125 \mathrm{eV}$ energy step. All the spectra were referenced to the main C 1s peak of the carbon atoms, which was assigned a value of $284.8 \mathrm{eV}$. The spectra were analysed using MultiPak v8.1c software (Ulvac-Phi Inc., Kanagawa, Japan, 2006) from Physical Electronics, which was supplied with the spectrometer. C1s spectra were fitted with a symmetrical Gauss-Lorentz function. A Shirley-type background subtraction was used. 


\subsection{Preparation of ER Fluids}

The corresponding powders (neat GO, GO-PHEMATMS-1, and GO-PHEMATMS-2) were sieved on sieves with a pore diameter of $45 \mu \mathrm{m}$ to obtain some representative portion for rheological investigation followed by drying in a vacuum oven at $60{ }^{\circ} \mathrm{C}$ for $24 \mathrm{~h}$ to eliminate potential water residues. The ER fluids were prepared by dispersing the corresponding particles in silicone oil (Lukosiol M200, Chemical Works Kolín, Kolín, Czech Republic; viscosity of $\eta_{\mathrm{c}}=194 \mathrm{mPa} \cdot \mathrm{s}$, conductivity of $\sigma_{\mathrm{c}} \approx 10^{-11} \mathrm{~S} \cdot \mathrm{cm}^{-1}$ ) in a $5 \mathrm{wt} . \%$ concentration. Before each experiment, the as-prepared system was first thoroughly stirred with a glass stick for approximately $5 \mathrm{~min}$ and then sonicated for $1 \mathrm{~min}$ to ensure the homogeneous distribution of the particles within the system.

\subsection{Electrorheological Measurements}

To prove the ER activity of particles under investigation, the steady shear measurements in a controlled shear rate mode were performed using a Bohlin CVOR 150 rotational rheometer (Malvern Instruments, Worchestershire, UK) with a parallel-plate geometry (diameter of $40 \mathrm{~mm}$, gap of $0.5 \mathrm{~mm}$ ). The external electric fields of a $0.5-2.5 \mathrm{kV} \cdot \mathrm{mm}^{-1}$ strength were generated using a DC TREEK 668B high-voltage source (TREK, USA). The experiment protocol was as follows: (i) Shearing the systems for $60 \mathrm{~s}$ at a shear rate of $50 \mathrm{~s}^{-1}$ either to homogeneously distribute the particles after the gap filling or to destroy residual chain-like structures after the previous measurement at a non-zero electric field strength, $E$; (ii) application of the external electric field of a given strength for $60 \mathrm{~s}$ before shearing to provide sufficient time for particles to organize themselves into stable, internal structures within the system; (iii) the shearing stage in the shear rate range of $0.1-300 \mathrm{~s}^{-1}$ with 5 points/decade in a logarithmic scaling. On/off cycle measurements were performed at a shear rate of $0.1 \mathrm{~s}^{-1}$ and $1.5 \mathrm{kV} \mathrm{mm}^{-1}$. One on/off cycle lasts $600 \mathrm{~s}$.

\subsection{Dielectric Measurements}

Dielectric relaxation spectroscopy of the corresponding ER fluids using a high-precision impedance analyser Novocontrol Concept 51 (Novocontrol, Montabaur, Germany) in a broad frequency range of $5-10^{5} \mathrm{~Hz}$ was applied as another method for the evaluation of ER activity. As the relaxation mechanism of ER fluids can be affected by temperature $[27,28]$, it should be noted that the characterization was performed at $25^{\circ} \mathrm{C}$.

\subsection{Sedimentation STABILITY}

The sedimentation stability measurement reflecting particles' compatibility with the carrier liquid was investigated for $5 \mathrm{wt}$.\% ER fluids via visual observation and evaluated by applying the sedimentation ratio concept (defined as the height of the particle-rich phase relative to the total system height), and its development in time.

\section{Results and Discussion}

The presence of PHEMATMS chains onto GO particles was confirmed via several independent techniques. First, the morphological aspect of the polymer grafted onto the GO particles was investigated using TEM images (Figure 1). This analysis evidently confirmed the 2D shape and well-exfoliated structure of individual GO sheets (Figure 1a). After the grafting of PHEMATMS with molar masses of $12600 \mathrm{~g} / \mathrm{mol}$ and $20400 \mathrm{~g} / \mathrm{mol}$ for GO-PHEMATMS-1 and GO-PHEMATMS-2 (Figure 2), with a dispersity of 1.19 and 1.13, respectively, from the GO surface, the sheet-like structure of $\mathrm{GO}$ was retained and the polymer formed a compact layer onto the GO surface (Figure 1b). A darker shade was observed for GO-PHEMATMS-2, i.e., for GO modified with higher molar mass PHEMATMS chains (Figure 1c). 


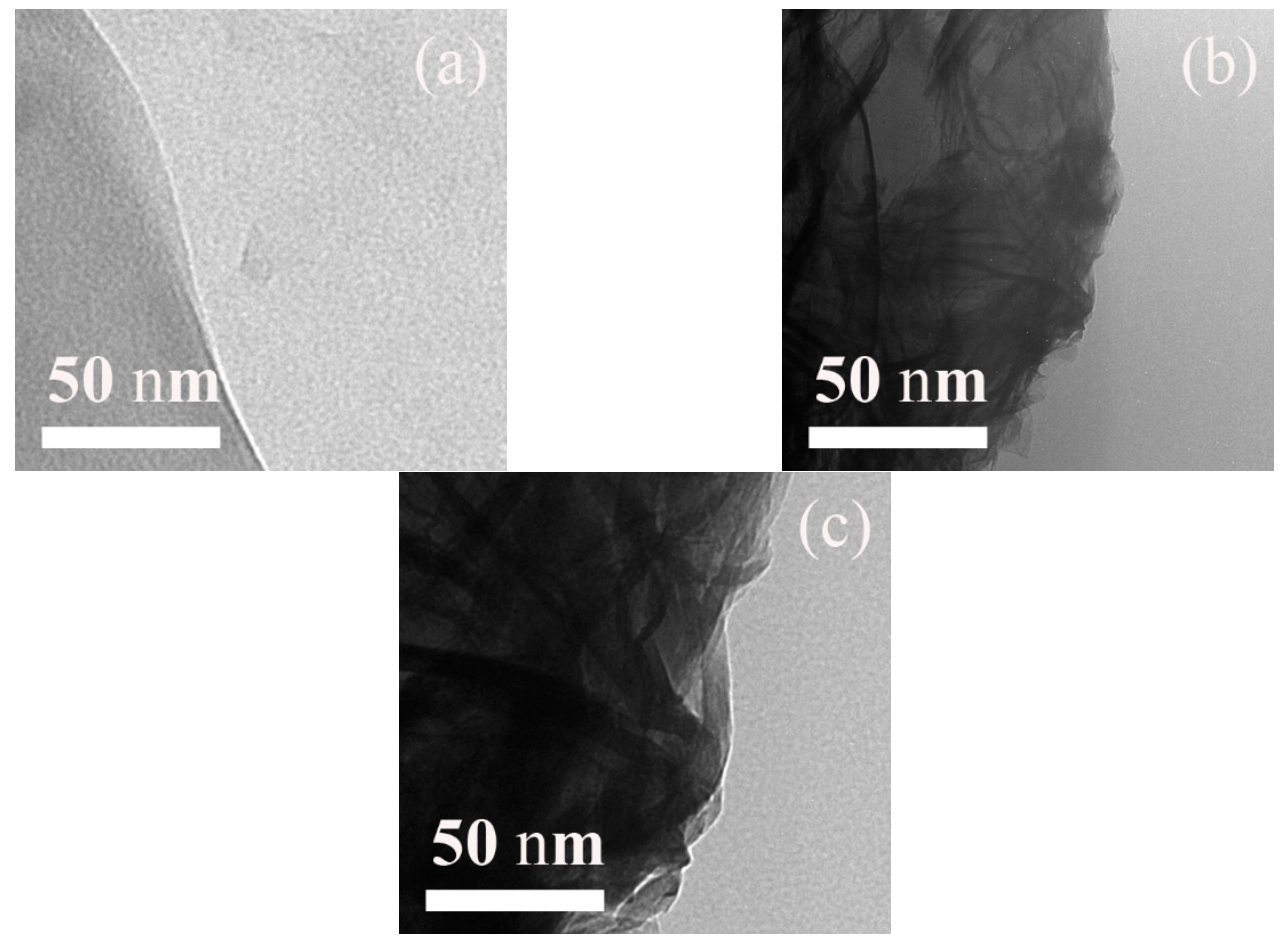

Figure 1. TEM images of unmodified GO (a), GO-PHEMATMS-1 (b), and GO-PHEMATMS-2 (c) particles.

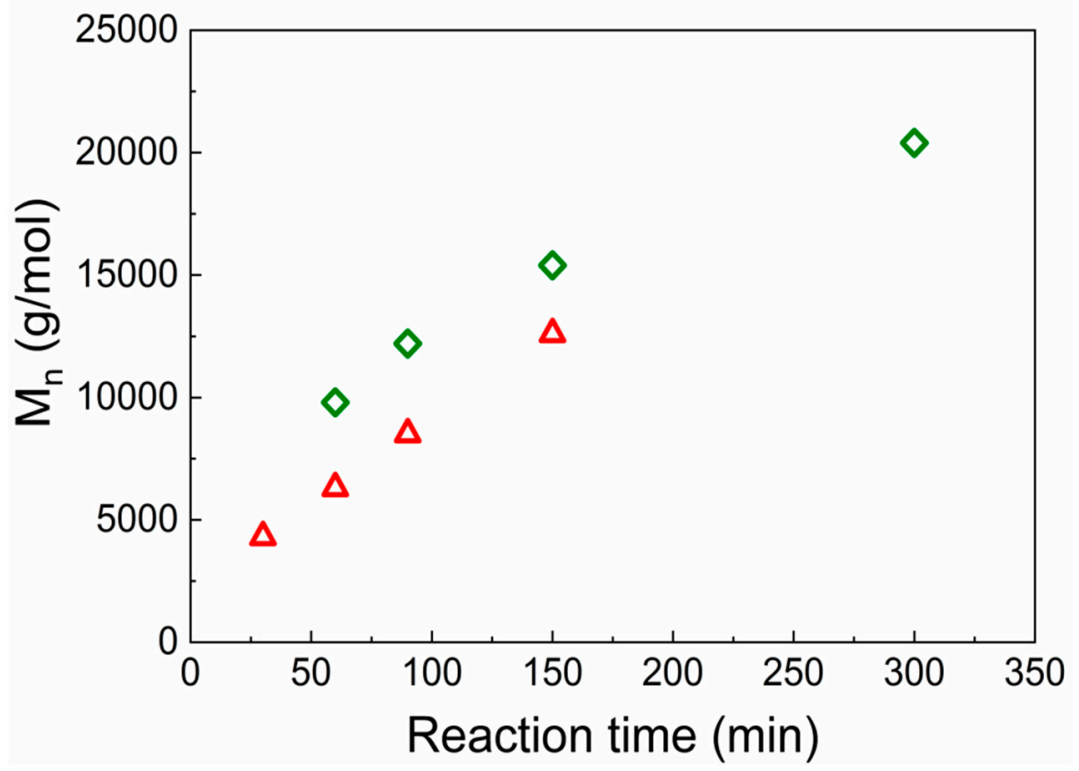

Figure 2. Evolution of the $M_{\mathrm{n}}$ versus the time of preparation of GO-PHEMATMS-1 $(\triangle)$ and GO-PHEMATMS-2 $(\diamond)$.

The chemical aspect of GO particles grafted with PHEMATMS chains was monitored using FTIR analysis (Figure 3). As expected, the FTIR spectrum of the original GO is in good agreement with those from previous works: A peak from the epoxy groups at around $823 \mathrm{~cm}^{-1}$, a broad peak from the $\mathrm{OH}$ groups at around $3500 \mathrm{~cm}^{-1}$, and a peak from the carboxyl and carbonyl groups at around $1700 \mathrm{~cm}^{-1}$ are all clearly visible, confirming the successful oxidation of graphite powder [25]. The successful grafting by PHEMATMS was confirmed by observation of new absorption bands corresponding to the PHEMATMS. A characteristic sharp peak at around $1726 \mathrm{~cm}^{-1}$ reflects the stretching vibration of $-\mathrm{C}=\mathrm{O}$ from the ester groups, and the broad absorption peak composed of individual sub-peaks at 
wavenumbers of around $1253 \mathrm{~cm}^{-1}, 1153 \mathrm{~cm}^{-1}$, and $1069 \mathrm{~cm}^{-1}$ indicates the presence of $-\mathrm{Si}-\mathrm{CH}_{3}$, $-\mathrm{C}-\mathrm{O}-$, and $-\mathrm{Si}-\mathrm{O}-$ bonds, respectively.

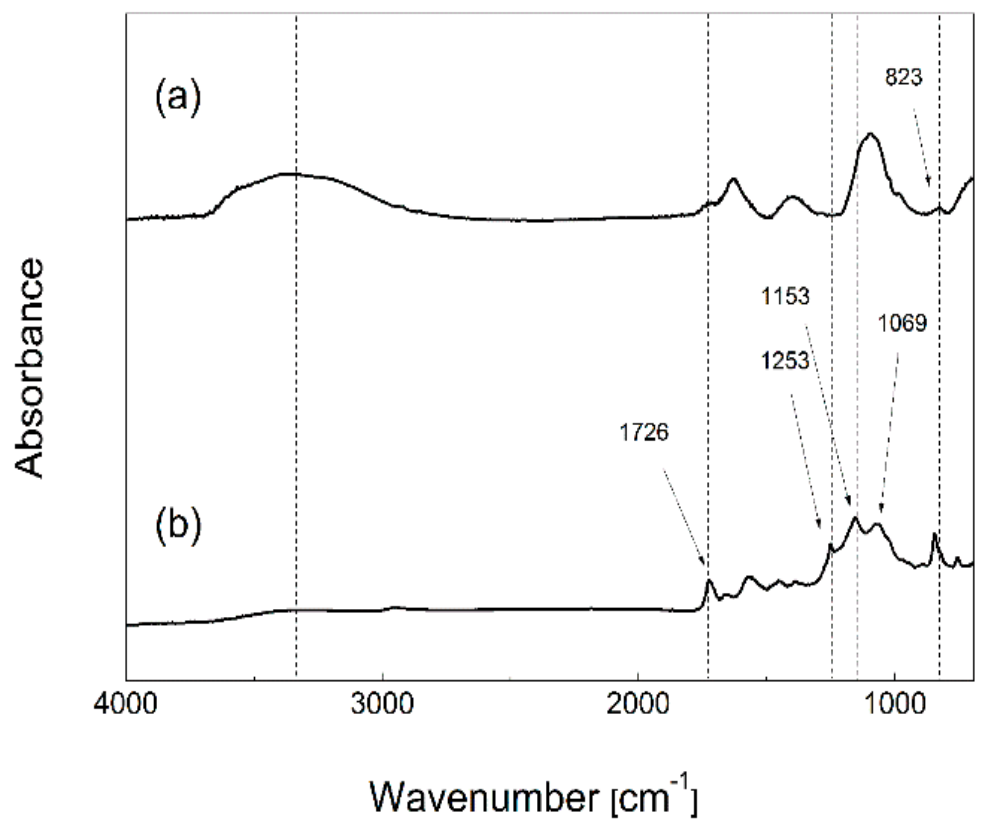

Figure 3. FTIR spectra for original GO (a) and GO-PHEMATMS (b) particles.

The surface initiated ATRP did not only provide grafting of GO particles with PHEMATMS chains, but also resulted in an increasing electric conductivity, $\sigma_{\mathrm{p}}$. While the $\sigma_{\mathrm{p}}$ for the original unmodified GO particles was only $1 \times 10^{-8} \mathrm{~S} \cdot \mathrm{cm}^{-1}$, i.e., close to the generally accepted limit of $\sigma_{\mathrm{p}}$ for sufficient ER activity [29], the $\sigma_{\mathrm{p}}$ of $4 \times 10^{-7}$ and $6 \times 10^{-6} \mathrm{~S} \cdot \mathrm{cm}^{-1}$ were determined for GO-PHEMATMS-1 and GO-PHEMATMS-2, respectively. This is in good agreement with our recent works, where we showed that controllably performed ATRP can provide systems with grafted polymer layers [26] with controlled thicknesses and precisely tuned $\sigma_{\mathrm{p}}[9]$.

To further prove that the partial and controllable reduction was performed, the Raman spectra of neat GO and GO-PHEMATMS-1 and GO-PHEMATMS-2 are shown in Figure 4. It can be seen that the ratio between the $\mathrm{sp}^{3}$ hybridization $(\mathrm{D}) / \mathrm{sp}^{2}$ hybridization $(\mathrm{G})$ peaks' intensities $\left(\mathrm{I}_{\mathrm{D}} / \mathrm{I}_{\mathrm{G}}\right)$ obtained after the SI-ATRP process increases as a confirmation of the partial reduction. Thus, a change from 0.90 to 1.08 and 1.11 was observed after grafting of PHEMATMS- 1 and PHEMATMS-2. It is also visible that the 2D sheet-like morphology of the GO created during oxidation remained nearly the same even after modification with various polymer chain lengths.
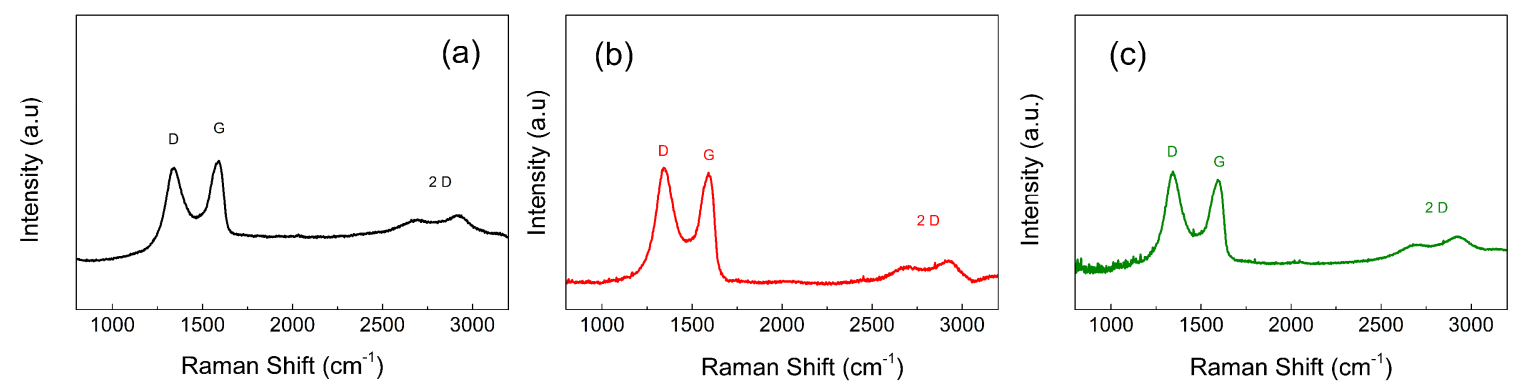

Figure 4. Raman spectra of the neat GO (a), GO-PHEMATMS-1 (b), and GO-PHEMATMS-2 (c).

The presence of the successful modification of the GO surface with PHEMATMS chains is confirmed by the appearance of new binding energies, the Si 2p and Si 2s peaks, from silyl-based monomer units. Moreover, the partial reduction of GO-polymer hybrids was confirmed by XPS spectra 
(Figure 5), where the carbon $(\mathrm{C})$ / oxygen $(\mathrm{O})$ ratio increased, and reached values of 2.00, 2.26, and 2.65 for neat GO, GO-PHEMATMS-1, and GO-PHEMATMS-2, respectively, indicating a lower amount of oxygen containing groups on the GO surface. Furthermore, the partial changes in the hybridization of $\mathrm{C} 1 \mathrm{~s}$ from sp3 to sp2 proved that the delocalized structure was restored and that the reduction took place. This evaluation is also summarized in Table 1.
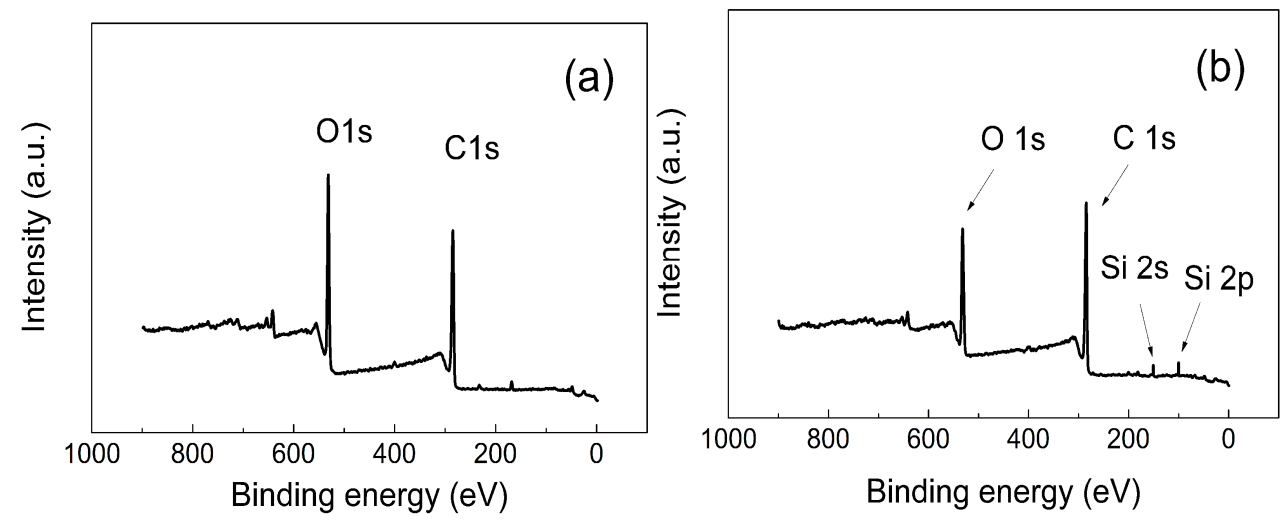

Figure 5. XPS spectra of the neat GO (a) and GO-PHEMATMS-1 particles (b).

Table 1. The surface chemical composition of the neat GO, GO-PHEMATMS-1, and GO-PHEMATMS-2 particles. All numbers are in atomic \%.

\begin{tabular}{ccccccccc}
\hline Sample Name & C1s & O1s & C1s sp2 & C1s sp3 & C1s C-O & C1s C=O & C1s O-C=O & C1s/O1s \\
\hline GO & 66.7 & 33.3 & 26.7 & 28.4 & 32.3 & 8.9 & 3.5 & 2.00 \\
GO-PHEMATMS-1 & 69.3 & 30.7 & 28.9 & 28.1 & 30.8 & 8.4 & 3.6 & 2.26 \\
GO-PHEMATMS-2 & 72.6 & 27.4 & 34.4 & 25.9 & 28.7 & 8.0 & 2.7 & 2.65 \\
\hline
\end{tabular}

The ER performance of 5 wt.\% systems based on unmodified GO particles and their modified analogues, GO-PHEMATMS-1 and GO-PHEMATMS-2, differing in both the thickness of grafted PHEMATMS and $\sigma_{\mathrm{p}}$, was compared both in the absence and in the presence of an external electric field. As can be seen in Figure 6, the ER fluid based on the unmodified GO particles exhibited a linear $\log -\log$ plot of the shear stress on the shear rate applied in the absence of the electric field, suggesting Newtonian flow behaviour characteristics. The ER fluids based on the PHEMATMS-grafted GO particles possessed a small divergence from the ideal flow behaviour. This deviation results from the improved interactions between the dispersed particles and carrier liquid, reflected in the improved sedimentation stability as will be discussed later. Briefly, the improvement in the mentioned interactions is caused by the decreased surface free energy of GO particles after their grafting with PHEMATMS chains. The particles with high surface free energy generally exhibit a lyophobic character, resulting in their aggregation into clusters in a time longer than that used in the performed rheological experiments. On the contrary, the particles with low surface free energy exhibit a lyophilic character, resulting in better wetting with the carrier liquid, similar to those observed in carbonyl iron-silicone oil systems [30]. Nonetheless, the deviation from the linearity in the case of PHEMATMS-grafted GO particles is negligible in the presence of an external electric field.

After the application of an external electric field, the ER phenomenon occurred for all three investigated systems, due to the formation of internal chain-like structures of polarized dispersed particles, reflecting the Bingham-like flow behaviour characteristics represented by the generation of a yield stress, $\tau_{y}$, defined as the minimal stress required to start the system flow again. Moreover, the value of $\tau_{\mathrm{y}}$ increased with the increase of E. Evidently from Figure 6, the ER effect (defined here as an increase in the Bingham-like flow behaviour at an applied $E$ ) increased with the grafting of GO particles with PHEMATMS chains and further with the thickness of the polymer layer. This can be a consequence of the enhancement of the $\sigma_{\mathrm{p}}$ and/or of the particles' polarizability as both contribute to the enhanced 
electro-responsive capabilities of the investigated systems. Moreover, the GO-PHEMATMS-2 based system (Figure 6c) exhibited values of shear stress of nearly $200 \mathrm{~Pa}$ at $3 \mathrm{kV} / \mathrm{mm}$, which is the highest value received for GO-based systems up to this date at such low particle concentrations (the following references summarize the GO-based systems and their ER performance [22,31-34]).
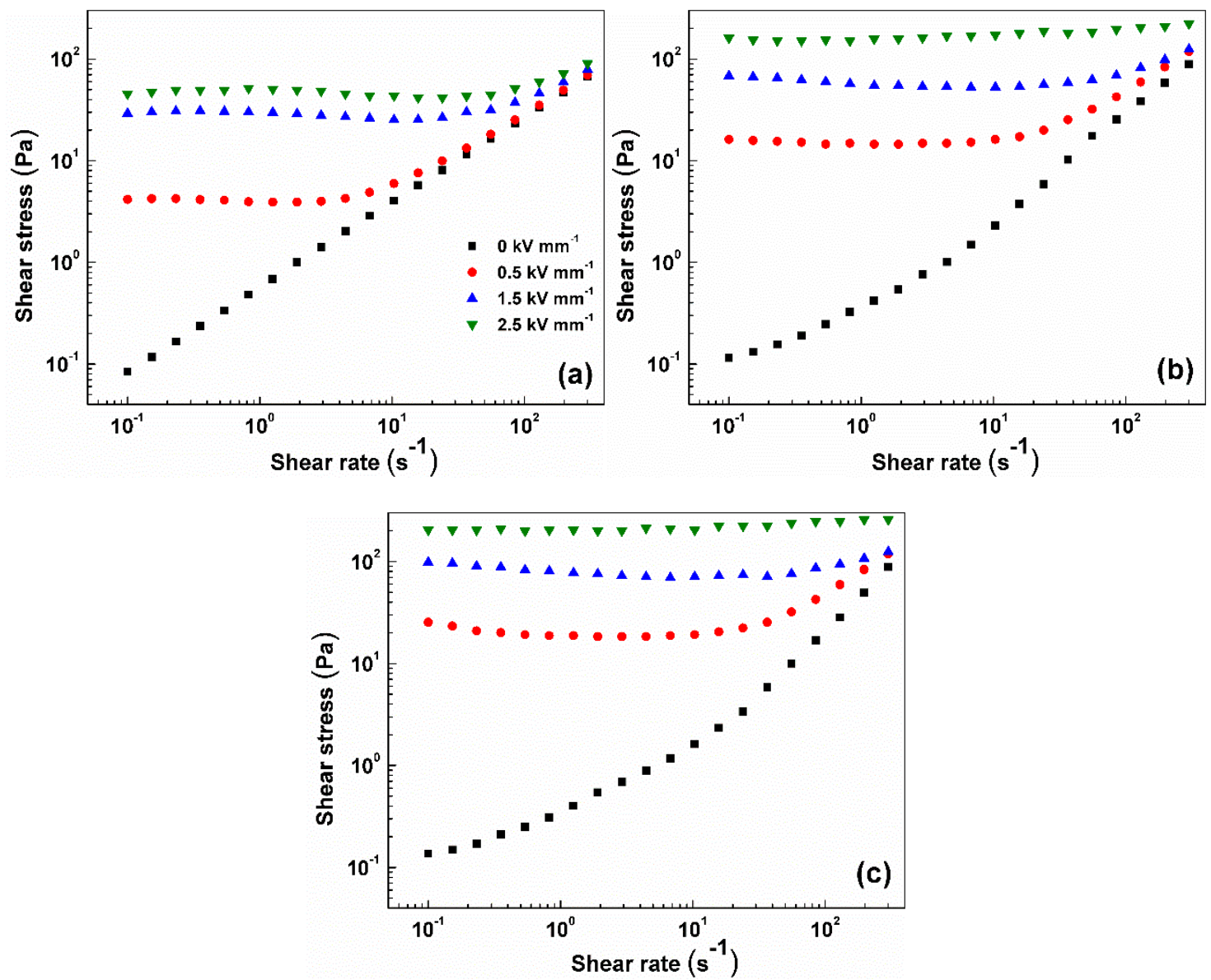

Figure 6. Dependence of the shear stress on the shear rate for $5 \mathrm{wt}$ \% ER fluids consisting of original GO (a), GO-PHEMATMS-1 (b), and GO-PHEMATMS-2 (c) particles in silicone oil M200. The electric field strengths $\left(\mathrm{kV} \cdot \mathrm{mm}^{-1}\right)$ : $0(\mathbf{\square}), 0.5(\bullet), 1.5(\mathbf{\Delta})$, and $2.5(\boldsymbol{\nabla})$.

As mentioned above, the stiffness of the internal chain-like structures formed under the external electric field can be proportionally expressed in the $\tau_{\mathrm{y}}$ value. Hence, Figure 7 depicts the dependence of $\tau_{\mathrm{y}}$ on the applied electric field to better illustrate the ER performance of the ER fluids under investigation. The $\tau_{y}$ values evaluated here were taken as shear stress values at very low shear rate $\left(0.1 \mathrm{~s}^{-1}\right)$ in Figure 6. It was proposed in electrorheology that this log-log dependence obeys the power law:

$$
\tau_{\mathrm{y}}=q \cdot E^{\alpha}
$$

where parameter $q$ represents the rigidity of the internal chain-like structures formed upon the applied electric field, and the value of parameter $\alpha$ should be around 1.5 or 2 for well-developed structures [35]. Evidently, from Table 2, all the investigated ER fluids had an $\alpha$ parameter of around 1.5. This, according to the theory, indicates that the conduction model is dominant factor for the ER performance of all three types of dispersed particles used within ER fluids [36]. Furthermore, the parameter, $q$, representing the rigidity of the internal chain-like structures, follows the dependence discussed in the ER effect evaluation part, with a significant increase of the rigidity when the polymer shell thickness is increased. 


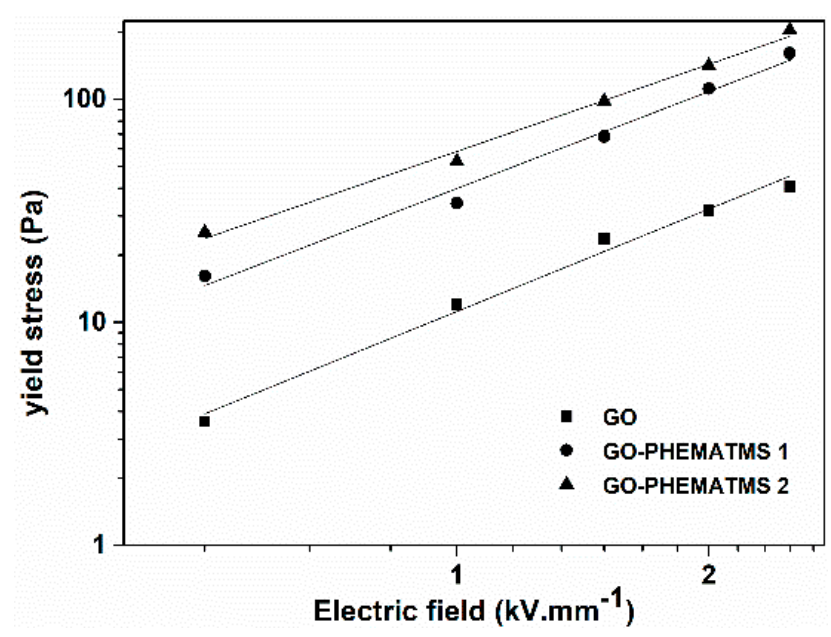

Figure 7. Values of the yield stress vs. the electric field strength for ER fluids consisting of various particles under investigation.

Table 2. Parameters of Equation (1) for $5 \mathrm{wt} \%$ ER fluids consisting of various particles under investigation.

\begin{tabular}{cccc}
\hline & GO & GO-PHEMATMS-1 & GO-PHEMATMS-2 \\
\hline$q$ & 11.2 & 39.8 & 58.4 \\
$\alpha$ & 1.5 & 1.45 & 1.44 \\
\hline
\end{tabular}

To prove the reproducibility of the phenomenon, five on/off cycles in which an electric field of $1 \mathrm{kV} \mathrm{mm}^{-1}$ was applied were performed (Figure 8). It can be clearly seen that the neat GO based suspensions show slight fluctuations during the on-state cycle and reach values of shear stress around $30 \mathrm{~Pa}$, indicating that the internal structure is not fully developed. However, both the suspensions based on GO-PHEMATMS show a stable behaviour during the on-state regime as a consequence of the well-developed internal structures. This behaviour is also supported by the fact that the shear stresses are $60 \mathrm{~Pa}$ and nearly $100 \mathrm{~Pa}$, for GO-PHEMATMS-1 and GO-PHEMATMS-2, respectively, when an electric field of $1.5 \mathrm{kV} \mathrm{mm}^{-1}$ was applied.

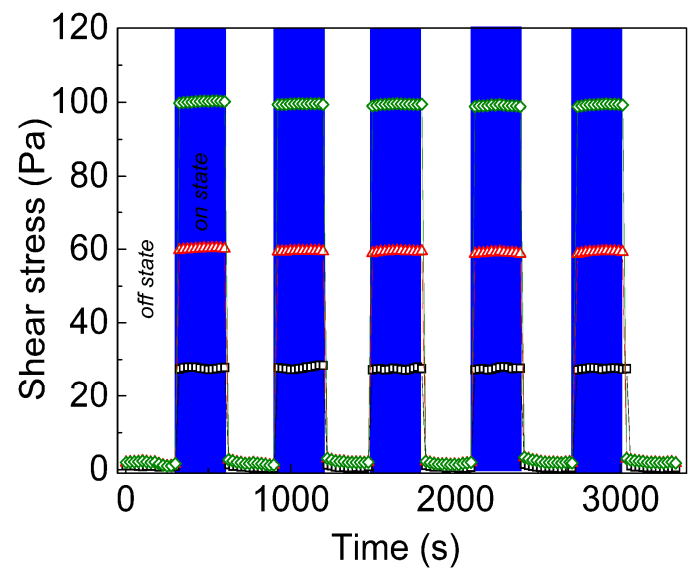

Figure 8. On/off cycles, showing the change of the shear stress for unmodified GO ( $\square$ ), GO-PHEMATMS-1 $(\triangle)$, and GO-PHEMATMS-2 $(\diamond)$ silicone oil suspensions during time in the absence and in the presence of an external electric field of $1.5 \mathrm{kV} \mathrm{mm}^{-1}$.

The suitability of the particles involved in this study for ER application was further confirmed via dielectric spectroscopy measurement. The ER effect generally stems from the particles' interfacial 
polarization in the presence of the external electric field. From an investigation of the registered relaxations in the dielectric spectra (Figure 9), the relaxation time, $t_{\text {rel }}$, and dielectric relaxation strength, $\Delta M^{\prime}$, can be obtained using the well-known Havriliak-Negami model [33] in its modified form [37]:

$$
\begin{gathered}
M_{H N}^{*}(\omega)=M_{\infty}^{\prime}+\frac{\Delta M^{\prime}}{\left(1+\left(i \omega \cdot t_{r e l}\right)^{\alpha}\right)^{\beta}} \\
M^{*}=M^{\prime}-i M^{\prime \prime}
\end{gathered}
$$

where $M^{*}$ is the complex dielectric modulus, $M_{\infty}^{\prime}$ is the unrelaxed (high frequency) dielectric modulus value, $i$ is the complex unit, and $M^{\prime}$ and $M^{\prime \prime}$ are the elastic and loss dielectric modulus, respectively. The $\omega$ is the angular frequency $(=2 \pi f)$.

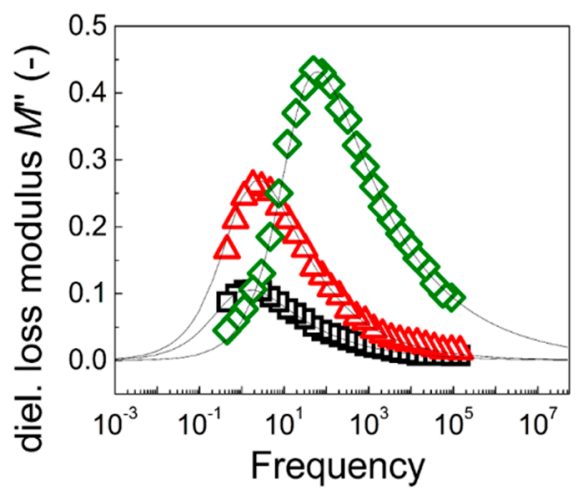

Figure 9. Dielectric loss moduli, $M^{\prime \prime}$, dependence on frequency for $5 \mathrm{wt}$ \% ER fluids consisting of unmodified GO $(\square)$, GO-PHEMATMS-1 $(\triangle)$, and GO-PHEMATMS-2 $(\diamond)$ particles in silicone oil M200.

As can be seen in Table 3, both parameters relevant for the efficient ER effect have the expected values. Indeed, $t_{\text {rel }}$ (located for all the investigated ER fluids within the frequency range of interfacial polarization) decreased, causing a faster response by the application of the external electric field, and the dielectric relaxation strength increased with the grafting of GO particles and with the higher molar mass of the polymer, due to both the better compatibility and the enhanced electric conductivity, and finally with the higher molar mass of the polymer. Therefore, the dielectric relaxation spectroscopy absolutely confirmed the results obtained in the ER experiments.

Table 3. Parameters of the Havriliak-Negami model, obtained after fitting related to the ER efficiency of the $5 \mathrm{wt}$.\% ER fluids consisting of the various particles under investigation.

\begin{tabular}{ccccccc}
\hline & $\boldsymbol{\Delta}^{\prime}$ & $\boldsymbol{M}_{\boldsymbol{\infty}}^{\prime}$ & $\boldsymbol{M}^{\boldsymbol{*}}{ }_{\mathbf{H N}}$ & $\boldsymbol{t}_{\text {rel }}[\mathbf{s}]$ & $\boldsymbol{\alpha}$ & $\boldsymbol{\beta}$ \\
\hline GO & 0.59 & 2.74 & 3.33 & 0.28 & 0.75 & 0.43 \\
GO-PHEMATMS_1 & 1.13 & 3.34 & 4.47 & 0.021 & 0.76 & 0.46 \\
GO-PHEMATMS_2 & 2.11 & 3.86 & 5.97 & 0.002 & 0.81 & 0.31 \\
\hline
\end{tabular}

The grafting of GO particles with PHEMATMS also positively influenced the stability of the investigated ER fluids against sedimentation. Both PHEMATMS-grafted GO systems exhibited a significantly higher sedimentation stability, expressed as the sedimentation ratio vs time dependence, than that based on the unmodified GO particles (Figure 10). The stability was more pronounced for GO-PHEMATMS-2, i.e., GO grafted with longer polymer chains (higher thickness of the polymer shell) probably due to the more lyophilic character of these particles causing their repulsion, which, as a result, eliminates particles aggregation. Moreover, as can be seen from the Figure 10 inset, the contact angle measurements showed that the mentioned grafting also influenced the wettability with contact angle values of $49.9^{\circ} \pm 3.2^{\circ}, 26.3^{\circ} \pm 2.7^{\circ}$, and $24.9^{\circ} \pm 1.4^{\circ}$, for neat GO, GO-PHEMATMS- 1 , and 
GO-PHEMATMS-2, respectively. All these findings significantly confirm the enhanced compatibility between the GO particles and the carrier liquid.

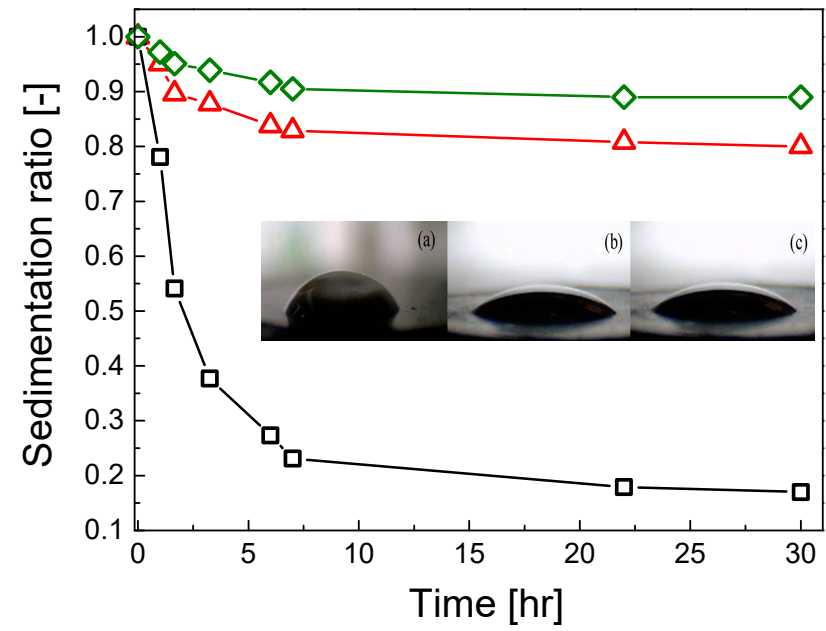

Figure 10. Sedimentation ratio as a function of time for $5 \mathrm{wt}$ \% ER fluids consisting of original GO ( $\square$ ), GO-PHEMATMS-1 $(\triangle)$, and GO-PHEMATMS-2 $(\diamond)$ particles in silicone oil M200.

\section{Conclusions}

A simple, single-step method of simultaneous grafting of the polymer layer and increasing the electric conductivity of the low conducting GO particles was used to prepare PHEMATMS-modified GO. The successful grafting with the silyl-based polymer was confirmed by TEM and FTIR spectroscopy. The parameters of the PHEMATMS chains were elucidated using the GPC and NMR technique, showing molar masses $12600 \mathrm{~g} / \mathrm{mol}$ and $20400 \mathrm{~g} / \mathrm{mol}$ for GO-PHEMATMS-1 and GO-PHEMATMS-2, respectively, while narrow polydispersity indexes of 1.19 and 1.13, respectively, were obtained. Electrorheological performance was significantly influenced by the mentioned grafting. The yield stress of $200 \mathrm{~Pa}$, determined for the system containing GO-PHEMATMS-2 at $2.5 \mathrm{kV} \mathrm{mm}^{-1}$, is uniquely high and has not been seen for GO-based systems at such low particle concentrations so far. The conductivity mechanism of the chain-like structure formation was consistent with previously published ones and the dielectric properties, together with the enhanced particle conductivity, significantly contributed to the improved ER capability. The reproducibility of the phenomenon was investigated by fiveon/off cycles and it was shown that GO-PHEMATMS based suspensions do not exhibit any fluctuation during the on-state and show shear stresses of $60 \mathrm{~Pa}$ and $100 \mathrm{~Pa}$ at $1.5 \mathrm{kV}$ $\mathrm{mm}^{-1}$ for GO-PHEMATMS-1 and GO-PHEMATMS-2, respectively. Finally, based on the contact angle results between the particles and the carrier liquid varying from $49.9^{\circ}$ for unmodified GO down to $26.3^{\circ}$ and $24.9^{\circ}$ for PHEMATMS-grafted GOs, it was demonstrated that a more hydrophobic character was obtained and thus the later systems exhibited an enhanced sedimentation stability, and provided a very promising system for various applications in electrorheology.

Author Contributions: M.I., M.M., and J.M. conceived and designed the experiments; E.K., J.O., M.I. and M.M. performed the experiments; E.K., M.I., J.O. and M.M. analyzed the data; E.K. and M.S. was responsible for designing, performing and discussing of contact angle and sedimentation stability properties. E.K., M.I., M.M., M.S. and J.M. wrote the paper.

Funding: Authors gratefully thanks to the Grant Agency of the Czech Republic (no. 16-20361Y) for financial support. This work was also supported by the Ministry of Education, Youth and Sports of the Czech Republic-program NPU I (LO1504). Authors M. I. and J. M. gratefully acknowledge to APVV-14-0891 and APVV-15-0545 for financial support. J. M. also gratefully acknowledges the Operational Program for Research, Development and Education, co-funded by the European Union, within the framework of project "International Mobility of Researchers of TBU in Zlín" (Reg. number: CZ.02.2.69/0.0/0.0/16_027/0008464) for financial support. M. I. thanks National Science Centre, Poland for the financial support through POLONEZ project number UMO-2016/23/P/ST5/02131.This project is carried out under POLONEZ program which has received funding 
from the European Unions's Horizon 2020 research and innovation programme under Marie Sklodowska-Curie grant agreement. No 665778 .

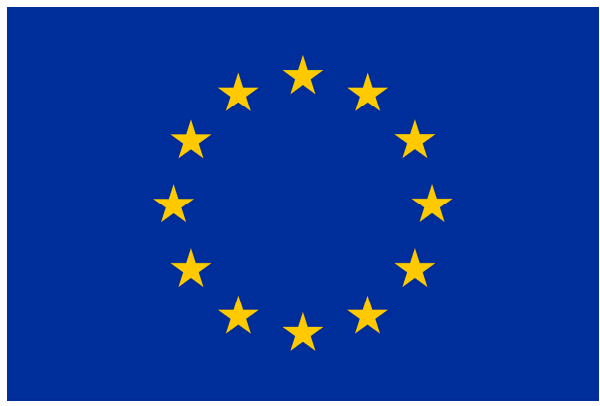

Conflicts of Interest: The authors declare no conflict of interest.

\section{References}

1. Jiang, J.L.; Tian, Y.; Meng, Y.G. Structure Parameter of Electrorheological Fluids in Shear Flow. Langmuir 2011, 27, 5814-5823. [CrossRef] [PubMed]

2. Stejskal, J.; Mrlik, M.; Plachy, T.; Trchova, M.; Kovarova, J.; Li, Y. Molybdenum and tungsten disulfides surface-modified with a conducting polymer, polyaniline, for application in electrorheology. React. Funct. Polym. 2017, 120, 30-37. [CrossRef]

3. Mrlik, M.; Cvek, M.; Osicka, J.; Moucka, R.; Sedlacik, M.; Pavlinek, V. Surface-initiated atom transfer radical polymerization from graphene oxide: A way towards fine tuning of electric conductivity and electro-responsive capabilities. Mater. Lett. 2018, 211, 138-141. [CrossRef]

4. Plachy, T.; Sedlacik, M.; Pavlinek, V.; Trchova, M.; Moravkova, Z.; Stejskal, J. Carbonization of aniline oligomers to electrically polarizable particles and their use in electrorheology. Chem. Eng. J. 2014, 256, 398-406. [CrossRef]

5. Plachy, T.; Mrlik, M.; Kozakova, Z.; Suly, P.; Sedlacik, M.; Pavlinek, V.; Kuritka, I. The Electrorheological Behavior of Suspensions Based on Molten-Salt Synthesized Lithium Titanate Nanoparticles and Their Core-Shell Titanate/Urea Analogues. ACS Appl. Mater. Interfaces 2015, 7, 3725-3731. [CrossRef] [PubMed]

6. Castro Neto, A.H.; Guinea, F.; Peres, N.M.R.; Novoselov, K.S.; Geim, A.K. The electronic properties of graphene. Rev. Mod. Phys. 2009, 81, 109-162. [CrossRef]

7. Marconcini, P.; Macucci, M. The k center dot $\mathrm{p}$ method and its application to graphene, carbon nanotubes and graphene nanoribbons: The Dirac equation. Riv. Nuovo Cimento 2011, 34, 489-584.

8. Kuila, T.; Bose, S.; Mishra, A.K.; Khanra, P.; Kim, N.H.; Lee, J.H. Chemical functionalization of graphene and its applications. Prog. Mater. Sci. 2012, 57, 1061-1105. [CrossRef]

9. Mrlik, M.; Ilcikova, M.; Plachy, T.; Pavlinek, V.; Spitalsky, Z.; Mosnacek, J. Graphene oxide reduction during surface-initiated atom transfer radical polymerization of glycidyl methacrylate: Controlling electro-responsive properties. Chem. Eng. J. 2016, 283, 717-720. [CrossRef]

10. Singh, V.; Joung, D.; Zhai, L.; Das, S.; Khondaker, S.I.; Seal, S. Graphene based materials: Past, present and future. Prog. Mater. Sci. 2011, 56, 1178-1271. [CrossRef]

11. Schniepp, H.C.; Li, J.L.; McAllister, M.J.; Sai, H.; Herrera-Alonso, M.; Adamson, D.H.; Prud'homme, R.K.; Car, R.; Saville, D.A.; Aksay, I.A. Functionalized single graphene sheets derived from splitting graphite oxide. J. Phys. Chem. B 2006, 110, 8535-8539. [CrossRef] [PubMed]

12. Mianehrow, H.; Moghadam, M.H.M.; Sharif, F.; Mazinani, S. Graphene-oxide stabilization in electrolyte solutions using hydroxyethyl cellulose for drug delivery application. Int. J. Pharm. 2015, 484, $276-282$. [CrossRef] [PubMed]

13. Lonkar, S.P.; Deshmukh, Y.S.; Abdala, A.A. Recent advances in chemical modifications of graphene. Nano Res. 2015, 8, 1039-1074. [CrossRef]

14. Zhang, W.L.; Liu, Y.D.; Choi, H.J.; Kim, S.G. Electrorheology of Graphene Oxide. ACS Appl. Mater. Interfaces 2012, 4, 2267-2272. [CrossRef] [PubMed]

15. Dreyer, D.R.; Park, S.; Bielawski, C.W.; Ruoff, R.S. The chemistry of graphene oxide. Chem. Soc. Rev. 2010, 39, 228-240. [CrossRef] [PubMed] 
16. Zhang, W.L.; Choi, H.J. Graphene oxide based smart fluids. Soft Matter 2014, 10, 6601-6608. [CrossRef] [PubMed]

17. Hui, C.M.; Pietrasik, J.; Schmitt, M.; Mahoney, C.; Choi, J.; Bockstaller, M.R.; Matyjaszewski, K. Surface-Initiated Polymerization as an Enabling Tool for Multifunctional (Nano-)Engineered Hybrid Materials. Chem. Mat. 2014, 26, 745-762. [CrossRef]

18. Hwang, B.; Lee, J.S. Recent Advances in Memory Devices with Hybrid Materials. Adv. Electron. Mater. 2019, 5, 22. [CrossRef]

19. Zhang, X.; Zhang, J.Y.; Chen, Y.; Cheng, K.; Yan, J.; Zhu, K.; Ye, K.; Wang, G.L.; Zhou, L.M.; Cao, D.X. Freestanding 3D Polypyrrole@reduced graphene oxide hydrogels as binder-free electrode materials for flexible asymmetric supercapacitors. J. Colloid Interface Sci. 2019, 536, 291-299. [CrossRef] [PubMed]

20. Wang, X.D.; Yu, K.X.; An, R.; Han, L.L.; Zhang, Y.L.; Shi, L.Y.; Ran, R. Self-assembling GO/modified HEC hybrid stabilized pickering emulsions and template polymerization for biomedical hydrogels. Carbohydr. Polym. 2019, 207, 694-703. [CrossRef] [PubMed]

21. Osicka, J.; Ilcikova, M.; Mrlik, M.; Minarik, A.; Pavlinek, V.; Mosnacek, J. The Impact of Polymer Grafting from a Graphene Oxide Surface on Its Compatibility with a PDMS Matrix and the Light-Induced Actuation of the Composites. Polymers 2017, 9, 14. [CrossRef]

22. Yin, J.B.; Wang, X.X.; Chang, R.T.; Zhao, X.P. Polyaniline decorated graphene sheet suspension with enhanced electrorheology. Soft Matter 2012, 8, 294-297. [CrossRef]

23. Yin, J.B.; Chang, R.T.; Shui, Y.J.; Zhao, X.P. Preparation and enhanced electro-responsive characteristic of reduced graphene oxide/polypyrrole composite sheet suspensions. Soft Matter 2013, 9, 7468-7478. [CrossRef]

24. Li, Y.Z.; Guan, Y.Q.; Liu, Y.; Yin, J.B.; Zhao, X.P. Highly stable nanofluid based on polyhedral oligomeric silsesquioxane-decorated graphene oxide nanosheets and its enhanced electro-responsive behavior. Nanotechnology 2016, 27, 11. [CrossRef] [PubMed]

25. Mrlik, M.; Ilcikova, M.; Plachy, T.; Moucka, R.; Pavlinek, V.; Mosnacek, J. Tunable electrorheological performance of silicone oil suspensions based on controllably reduced graphene oxide by surface initiated atom transfer radical polymerization of poly(glycidyl methacrylate). J. Ind. Eng. Chem. 2018, 57, 104-112. [CrossRef]

26. Ilcikova, M.; Mrlik, M.; Spitalsky, Z.; Micusik, M.; Csomorova, K.; Sasinkova, V.; Kleinova, A.; Mosnacek, J. A tertiary amine in two competitive processes: Reduction of graphene oxide vs. catalysis of atom transfer radical polymerization. RSC Adv. 2015, 5, 3370-3376. [CrossRef]

27. Zhang, W.L.; Park, B.J.; Choi, H.J. Colloidal graphene oxide/polyaniline nanocomposites and its electrorheology. Chem. Commun. 2010, 46, 5596-5598. [CrossRef] [PubMed]

28. Plachy, T.; Sedlacik, M.; Pavlinek, V.; Stejskal, J.; Graca, M.P.; Costa, L.C. Temperature-dependent electrorheological effect and its description with respect to dielectric spectra. J. Intell. Mater. Syst. Struct. 2016, 27, 880-886. [CrossRef]

29. Plachy, T.; Sedlacik, M.; Pavlinek, V.; Stejskal, J. The observation of a conductivity threshold on the electrorheological effect of p-phenylenediamine oxidized with p-benzoquinone. J. Mater. Chem. C 2015, 3, 9973-9980. [CrossRef]

30. Sedlacik, M.; Pavlinek, V. A tensiometric study of magnetorheological suspensions' stability. RSC Adv. 2014, 4, 58377-58385. [CrossRef]

31. Hong, J.Y.; Lee, E.; Jang, J. Electro-responsive and dielectric characteristics of graphene sheets decorated with $\mathrm{TiO}_{2}$ nanorods. J. Mater. Chem. A 2013, 1, 117-121. [CrossRef]

32. Hu, H.T.; Wang, X.B.; Wang, J.C.; Liu, F.M.; Zhang, M.; Xu, C.H. Microwave-assisted covalent modification of graphene nanosheets with chitosan and its electrorheological characteristics. Appl. Surf. Sci. 2011, 257, 2637-2642. [CrossRef]

33. Ilcikova, M.; Mrlik, M.; Babayan, V.; Kasak, P. Graphene oxide modified by betaine moieties for improvement of electrorheological performance. RSC Adv. 2015, 5, 57820-57827. [CrossRef]

34. Chen, P.; Cheng, Q.; Wang, L.M.; Liu, Y.D.; Choi, H.J. Fabrication of dual-coated graphene oxide nanosheets by polypyrrole and poly (ionic liquid) and their enhanced electrorheological responses. J. Ind. Eng. Chem. 2019, 69, 106-115. [CrossRef]

35. Davis, L.C. Time-dependent and nonlinear effects in electrorheological fluids. J. Appl. Phys. 1997, 81, 1985-1991. [CrossRef] 
36. Zhang, K.; Liu, Y.D.; Jhon, M.S.; Choi, H.J. Generalized yield stress equation for electrorheological fluids. J. Colloid Interface Sci. 2013, 409, 259-263. [CrossRef] [PubMed]

37. Moucka, R.; Sedlacik, M.; Cvek, M. Dielectric properties of magnetorheological elastomers with different microstructure. Appl. Phys. Lett. 2018, 112, 4. [CrossRef]

(c) (

(C) 2019 by the authors. Licensee MDPI, Basel, Switzerland. This article is an open access article distributed under the terms and conditions of the Creative Commons Attribution (CC BY) license (http:/ / creativecommons.org/licenses/by/4.0/). 